\title{
Additional Thoughts on Drug Shortages
}

I applaud and support Vaillancourt's editorial on the topic of drug shortages in a recent issue of the Canadian Journal of Hospital Pharmacy. ${ }^{1}$ The topic and the editorialist's comments resonated with my experience. Each year, I find myself stating that "this is worst year I've ever seen for back orders", only to have an even worse situation arise the following year.

I absolutely agree that a new model of "strategic sourcing" must be undertaken, whereby the contract for drug supply is split between 2 or more vendors. Although this approach is not needed for all contracts, it should certainly be applied for critical medications. As pharmacists, we have a leadership role in advocating for this type of model with our purchasing groups or senior hospital administrators. The alternative, in the event of a shortage, is that patient care may be compromised. In addition, there is a human resource cost associated with trying to find alternative sources. Finally, even if an alternative can be found, it is often more expensive. In addition to Vaillancourt's vaccine example, the strategic sourcing model is also being used successfully by Canadian Blood Services for IV immunoglobulin and was previously used for plasma expanders.

Unfortunately, the process has been slow to gain acceptance among vendors, who anticipate reduced sales, and among hospitals, who anticipate increased acquisition costs. Although cost savings are important, focusing solely on the lowest acquisition cost can be short-sighted if continuity of supply is not maintained.

We should also be advocating for the concept of manufacturing redundancy. In other words, a company's manufacturing plants should have the ability to maintain some production of at least critical or single-source products if a failure occurs at the company's main production site. However, such redundancy is expensive, and most companies do not have this ability unless it is required. In fact, I know of only one company that has production redundancy built into its system.

Finally, we should be advocating for the federal government, specifically Health Canada, to take a greater role in the prevention and monitoring of medication shortages. The Sandoz back-order situation has exposed many gaps in the exist- 
ing drug supply system. What is generally not known is that most of the decisions with respect to supplying the Canadian market were made by the parent company, Novartis International AG of Germany. In an example from another industry, the federal government blocked the $\$ 39$ billion takeover of Potash Corporation of Saskatchewan because it did not provide a net benefit to Canada. Who would have thought that the foreign takeover of a little known Canadian pharmaceutical company (Sabex) would have such repercussions in the future?

What is now apparent is that the previous models of supply and procurement require rethinking. It also appears that this issue may get worse before it gets better.

\section{Reference}

1. Vaillancourt R. Drug shortages: What can hospital pharmacists do? [editorial]. Can J Hosp Pharm 2012;65(3):175-176.

Jeff Chan, RPh, PharmD

Manager

Pharmacy Department

Thunder Bay Regional Health Sciences Centre

Thunder Bay, Ontario 